THE COMPUTATION OF MOLECULAR ABUNDANCES AND OPACITIES IN THE ATMOSPHERES OF LATE-TYPE STARS

\author{
Christopher M. Sharp \\ $\mathrm{T}-4$ \\ Los Alamos National Laboratory \\ Los Alamos, NM 87545 \\ USA
}

\begin{abstract}
The presence of molecules in cool stellar atmospheres can dominate the opacity. Some calculations have been done with a carbonrich mixture of $\mathrm{C} / \mathrm{O}=2$, and the molecules $\mathrm{C}_{2}, \mathrm{CN}, \mathrm{HCN}$ and $\mathrm{C}_{2} \mathrm{H}_{2}$ are major sources of opacity due to their large abundances. New calculations with HCN and $\mathrm{C}_{2} \mathrm{H}_{2}$ show they can be important.
\end{abstract}

METHODS AND RESULTS

The calculation of models of late-type evolved stars with carbon-rich atmospheres depends on reliable values of the opacity, which are unfortunately difficult to obtain at low temperatures due to a large number of lines produced by several carbon-containing molecules.

The opacities calculated by Johnson (1982), with the bands of diatomic molecules being considered, appear to be too small for reasonable stellar models, and when some of these opacities were recalculated (Lucy et a1. 1985), it was found that larger opacities were indeed obtained. Here some calculations are repeated with $\mathrm{HCN}$ and $\mathrm{C}_{2} \mathrm{H}_{2}$.

As in Lucy et a1. (1985), the most carbon-rich of Johnson's mixtures is considered, with the carbon abundance enhanced to $C / O=2$ and with ${ }^{12} \mathrm{C} /{ }^{13} \mathrm{C}=10$, while keeping the other elements and their isotopes to solar abundance.

On the assumption of LTE, the abundance of electrons, ions, atoms, and molecules at a given value of $\mathrm{T}$ and $\rho$ are obtained by iterating the equations of ionization and dissociation for a mixture of the 22 most abundant elements and 52 diatomic and polyatomic molecules with isotopic variants. As before, the Rosseland opacity is obtained by using a grid of 300,000 frequencies on which the continuum and molecular bands are calculated on a line-by-line basis. The lines have Gaussian profiles broadened by thermal and an assumed turbulent velocity.

Bands of $\mathrm{HCN}$ and $\mathrm{C}_{2} \mathrm{H}_{2}$ are included by using the harmonic oscillator approximation to calculate the strengths of "hot" bands in a given sequence, when the strength of the first member of that sequence is known (Sharp, 1981); bands of diatomic molecules are calculated as before.

Preliminary results are given in Table I for Rosseland opacities with the same turbulent velocity as Lucy et al. 
TABLE I. Rosseland opacities in $\mathrm{cm}^{2} / \mathrm{g}$ for a mixture with $\mathrm{C} / 0=2$ at $\log \rho=-9$ and a turbulent velocity of $5 \mathrm{~cm} / \mathrm{s}$.

\section{Opacity Source}

Continuum alone

Con. + diatomic molecules

Con. + diatomic molecules + $\mathrm{HCN}$

Con. + diatomic molecules $+\mathrm{C}_{2} \mathrm{H}_{2}$

\section{$\underline{\log \bar{K}}$ at $\log T=3.3$}

$-5.56$

$-4.56$

$-3.56$

$-3.90$ $\underline{\log \bar{K} \text { at } \log \mathrm{T}=3.4}$

$-4.48$

$-2.31$

$-1.49$

$-1.95$

A plot of the harmonically-averaged opacity is shown in Fig. 1 with a turbulent velocity of $5 \mathrm{~km} / \mathrm{s}$ for $\mathrm{HCN}$ superimposed on a plot of diatomic bands with the continuum below. As can be seen, the effects of HCN and $\mathrm{C}_{2} \mathrm{H}_{2}$ can be very important in cool carbon-rich atmospheres, as they can fill gaps in the absorption left by the diatomic molecules.

I am grateful to Dr. S. P. Tarafdar for providing funds to attend Goa, and Dr. W. F. Huebner for useful comments. This work was carried out under the auspices of the US Department of Energy.

\section{REFERENCES}

Johnson, H.R., Ap. J. 260, 254 (1982).

Lucy, L.B., Robertson, J.A. and Sharp, C.M., European Southern Observatory Preprint No. 394 (1985), and submitted to Astro. Astroph.

Sharp, C.M., Ph.D. Thesis, University of St. Andrews (1981).

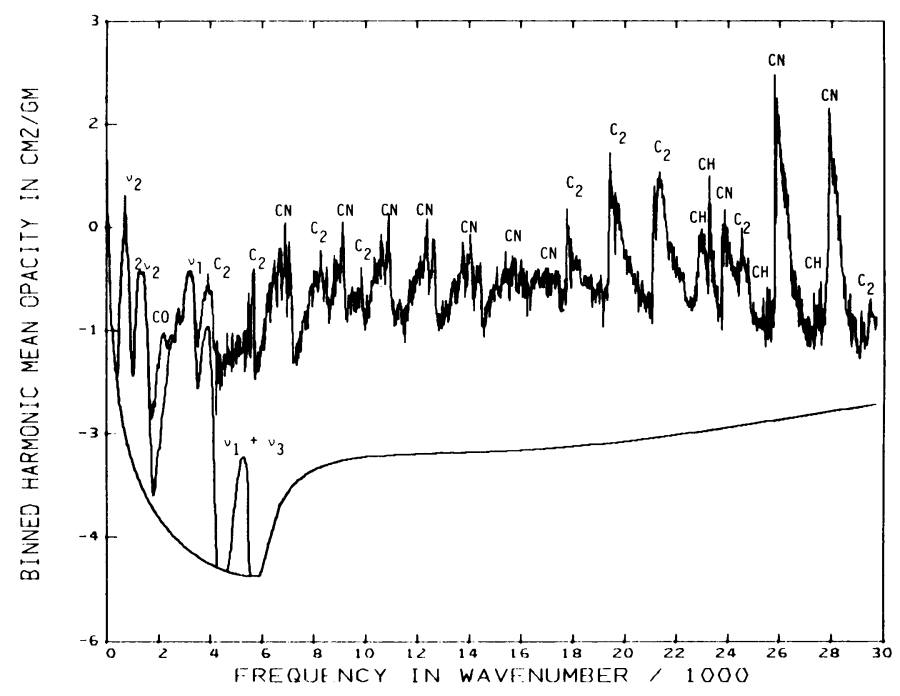

Figure 1. The harmonically-averaged absorption coefficient vs. wavenumber in $\mathrm{cm}^{-1}$ for $\log \mathrm{T}=3.4$ and $\log \rho=-9$, with bands of $\mathrm{HCN}$ being indicated. 